\title{
Generalized Israel Junction Conditions for a Fourth-Order Brane World
}

\author{
Adam Balcerzak and Mariusz P. Dạbrowsk闹 \\ Institute of Physics, University of Szczecin, Wielkopolska 15, 70-451 Szczecin, Poland.
}

(Dated: October 29, 2018)

\begin{abstract}
We discuss a general fourth-order theory of gravity on the brane. In general, the formulation of the junction conditions (except for Euler characteristics such as Gauss-Bonnet term) leads to the higher powers of the delta function and requires regularization. We suggest the way to avoid such a problem by imposing the metric and its first derivative to be regular at the brane, while the second derivative to have a kink, the third derivative of the metric to have a step function discontinuity, and no sooner as the fourth derivative of the metric to give the delta function contribution to the field equations. Alternatively, we discuss the reduction of the fourth-order gravity to the second-order theory by introducing an extra tensor field. We formulate the appropriate junction conditions on the brane. We prove the equivalence of both theories. In particular, we prove the equivalence of the junction conditions with different assumptions related to the continuity of the metric along the brane.

PACS numbers: $98.80 . \mathrm{Cq}$
\end{abstract}

\section{INTRODUCTION}

Brane universes have made great popularity during the last years 1, 2. However, it is remarkable that so far only the standard Einstein gravity, Gauss-Bonnet gravity [3, 4, 5, 6, 7, 8, 9] and, in general, Euler density gravity [10] on the brane have been considered in the literature [11]. These can be expressed by the general action ${ }^{1}$

$$
S=\int_{M} d^{D} x \sqrt{-g} \sum_{n} \kappa_{n} I^{(n)}+S_{b r a n e}+S_{m}
$$

where $I^{(n)}$ is the Euler density of the n-th order, $\kappa_{n}$ is an appropriate constant of the n-th order, $M$ is a $D$ dimensional manifold, $S_{\text {brane }}$ is the brane action and $S_{m}$ is the matter action. The lowest order Euler densities are: the cosmological constant $I^{(0)}=1$, the Ricci scalar $I^{(1)}=R$, and the Gauss-Bonnet density $I^{(2)}=R_{G B}=$ $R_{a b c d} R^{a b c d}-4 R_{a b} R^{a b}+R^{2}$ with appropriate constants $\kappa_{0}=-2 \Lambda\left(2 \kappa^{2}\right)^{-1}=-2 \Lambda / 16 \pi G, \kappa_{1}=\left(2 \kappa^{2}\right)^{-1}, \kappa_{2}=$ $\alpha\left(2 \kappa^{2}\right)^{-1}, \alpha=$ const. etc., $a, b, c=0,1, \ldots, D-3, D-$ $2, D[5]$.

In fact, in a general class of brane models based on an arbitrary combination of the higher-order curvature terms $f\left(R_{a b c d} R^{a b c d}, R_{a b} R^{a b}, R\right)$ the field equations are fourth-order. Because of that they are plagued by the higher power terms of the second derivative of the warp factor function $\sigma(y)=|y|$. This leads to a production of the higher powers of the delta function $\delta(y)$ which can make the field equations ambiguous. Among the general class, the models based on the Euler densities are unique in the sense that the higher powers of the second derivative of the warp factor $\partial^{2} \sigma(y) / \partial y^{2}$ exactly cancel in the

\footnotetext{
*Electronic address: abalcerz@wmf.univ.szczecin.pl

${ }^{\dagger}$ Electronic address: mpdabfz@sus.univ.szczecin.pl

${ }^{1}$ We use convention $(-++\ldots+)$ for the metric following Ref. [12.
}

field equations [11. One then is easily able to formulate appropriate junction conditions given first by Israel [13, 14].

The main objective of our paper will be the study of a general fourth-order theory of gravity on the brane [15]

$$
S=\chi^{-1} \int_{M} d^{D} x \sqrt{-g} f(X, Y, Z)+S_{\text {brane }}+S_{m}(\text { I. } 2)
$$

where $X=R, Y=R_{a b} R^{a b}, R_{a b c d} R^{a b c d}$ are curvature invariants, and $\chi$ is a constant. It includes the Euler density theories with the first Euler density being just $f(X, Y, Z)=\chi \kappa_{1} X=\chi \kappa_{1} R$ and the second Euler density being the Gauss-Bonnet term given by $f(X, Y, Z)=\chi \kappa_{2}\left(Z-4 Y+X^{2}\right)$ etc.

Up to our knowledge, the only non-Eulerian density cases were studied in Refs. [16] and 17. In Ref. 16] the fourth-order theory $f(X, Y, Z)=f(X)=f(R)$ was first reduced to the second-order theory, and then transformed into the Einstein theory. The junction conditions were then obtained, and they were obviously free from the problem of the powers of $\delta$-function contribution. On the other hand, in Ref. [17] the theories with the linear combination of the form $f(X, Y, Z)=a X^{2}+b Y+c Z$ $(a, b, c=$ const.) were considered and the junction conditions were obtained by the application of the appropriate Gibbons-Hawking boundary terms, again after transforming this theory to an equivalent second-order theory.

It is important to emphasize that the theories based on the functions of the Euler densities such as $f\left(I^{(n)}\right)$ are fourth-order. Among them the most popular are $f\left(I^{(1)}\right)=f(R)$ - the theories of the function of the first Euler density [18. In fact, the theories which are based on the function of the second Euler density $f\left(I^{(2)}\right)$ have also gained some interest recently [19, but they have not been studied on the brane yet.

Our paper is organized as follows. In Section II we discuss the main obstacle to formulate junction conditions for the fourth-order braneworld in a standard way which has been performed in the case of Euler densities. In Sec- 
tion III we make a proposal to formulate these junction conditions by imposing more regularity onto the metric tensor. Since it does not necessarily satisfy everybody's taste we present in Section IV an alternative approach. In this approach we transform our general fourth-order theory into a second-order theory by applying generalized Lagrange-multiplier approach [18, 19, 26]. This method was successful in obtaining the junction conditions in $f(R)$ theory [16] and in $f=a X^{2}+b Y+c Z$ theory [17]. In the Section $V$ we formulate the junction conditions for the equivalent second-order theory. Finally, in Section VI we give our conclusions.

\section{PROBLEM FORMULATING ISRAEL JUNCTION CONDITIONS IN A FOURTH-ORDER BRANE WORLD}

In order to discuss the problem let us begin with the standard D-dimensional brane theory [1] whose action is just (I.1) with only $\kappa_{1} \neq 0$, i.e.,

$$
S=\frac{1}{2 \kappa^{2}} \int_{M} d^{D} x \sqrt{-g} R+S_{\text {brane }}+S_{m}
$$

with the field equations

$$
G_{a}^{b}=R_{a}^{b}-(1 / 2) \delta_{a}^{b} R=\kappa^{2} T_{a}^{b},
$$

where the energy-momentum tensor is given by

$$
T_{a}{ }^{b}=T_{a}{ }^{b-} \Theta(-w)+T_{a}{ }^{b+} \Theta(w)+\delta(w) S_{a}{ }^{b},
$$

with $S_{a}{ }^{b}$ being the energy-momentum tensor on the brane, and $T_{a}{ }^{b \pm}$ are the energy-momentum tensors on the both sides of the brane (i.e., in the bulk).

For simplicity, let us assume that we work in Gaussian normal coordinates - i.e., that the D-dimensional metric is of the form $(\mu, \nu=0,1,2, \ldots, D-2 ; w=D)$

$$
d s^{2}=g_{a b} d x^{a} d x^{b}=\epsilon d w^{2}+h_{\mu \nu} d x^{\mu} d x^{\nu},
$$

where $\epsilon=\vec{n} \cdot \vec{n}=+1$ for a spacelike hypersurface, $\epsilon=-1$ for a timelike hypersurface, and $h_{a b}=g_{a b}-\epsilon n_{a} n_{b}$ is a projection tensor onto a $(D-1)$-dimensional hypersurface, $\vec{n}$ is the normal vector to the hypersurface. The extrinsic curvature in these coordinates is defined as

$$
K_{\mu \nu}=-\frac{1}{2} \frac{\partial h_{\mu \nu}}{\partial w}
$$

By the application of the Gauss-Codazzi equations 23.

$$
\begin{aligned}
R_{w \mu w \nu} & =\frac{\partial K_{\mu \nu}}{\partial w}+K_{\rho \nu} K_{\mu}^{\rho}, \\
R_{w \mu \nu \rho} & =\nabla_{\nu} K_{\mu \rho}-\nabla_{\rho} K_{\mu \nu}, \\
R_{\lambda \mu \nu \rho} & ={ }^{(D-1)} R_{\lambda \mu \nu \rho}+\epsilon\left[K_{\mu \nu} K_{\lambda \rho}-K_{\mu \rho} K_{\lambda \nu}\right](\text { II. } .8)
\end{aligned}
$$

one has the D-dimensional field equations in the form

$$
\begin{aligned}
G^{w}{ }_{w} & =-\frac{1}{2}{ }^{(D-1)} R+\frac{1}{2} \epsilon\left[K^{2}-\operatorname{Tr}\left(K^{2}\right)\right]=\kappa^{2} T_{w}^{w},(\text { II.9) } \\
G^{w}{ }_{\mu} & =\epsilon\left[\nabla_{\mu} K-\nabla_{\nu} K^{\nu}{ }_{\mu}\right]=\kappa^{2} T_{\mu}^{w}, \\
G^{\mu}{ }_{\nu} & ={ }^{(D-1)} G^{\mu}{ }_{\nu}+\epsilon\left[\frac{\partial K^{\mu}{ }_{\nu}}{\partial w}-\delta^{\mu}{ }_{\nu} \frac{\partial K}{\partial w}\right] \\
& +\epsilon\left[-K K^{\mu}{ }_{\nu}+\frac{1}{2} \delta^{\mu}{ }_{\nu} \operatorname{Tr}\left(K^{2}\right)+\frac{1}{2} \delta^{\mu}{ }_{\nu} K^{2}\right]=\kappa^{2} T^{\mu}{ }_{\nu} .
\end{aligned}
$$

The Israel junction conditions can be obtained by the integration of the field equations (II.9)-(II.11) in the limit $\lim _{w \rightarrow 0} \int_{-w}^{w}[3]$ and read as

$$
\begin{aligned}
\epsilon\left\{\left[K_{\nu}^{\mu}\right]-\delta_{\nu}^{\mu}[K]\right\} & =\kappa^{2} S^{\mu}{ }_{\nu}, \\
0 & =\kappa^{2} S^{w}{ }_{w}, \\
0 & =\kappa^{2} S^{w}{ }_{\mu},
\end{aligned}
$$

where $\left[K^{\mu}{ }_{\nu}\right] \equiv K^{\mu}{ }_{\nu}{ }^{-}-K^{\mu}{ }_{\nu}{ }^{-}$. In general, for any quantity $\Omega$, one defines $[\Omega]=\Omega^{+}-\Omega^{-}$, where $\Omega^{ \pm}$means that this quantity was calculated on the left-hand-side and on the right-hand-side of the brane, respectively.

The most important point is that these junction conditions are obtained provided we assume the following continuity conditions for the metric at $w=0$ [14]:

$$
\begin{aligned}
h_{\mu \nu}^{-} & =h_{\mu \nu}^{+}, \\
h_{\mu \nu, w}^{-} & \neq h_{\mu \nu, w}^{+}, \quad K_{\mu \nu}^{-} \neq K_{\mu \nu}^{+},
\end{aligned}
$$

which means that the metric is continuous at the brane but it has a kink, its first derivative has a step function discontinuity, and its second derivative gives the delta function contribution. In other words:

$$
\begin{aligned}
h_{\mu \nu}(w) & =h_{\mu \nu}^{-}(w) \theta(-w)+h_{\mu \nu}^{+}(w) \theta(w), \\
\frac{\partial h_{\mu \nu}^{-}}{\partial w} & =\frac{\partial h_{\mu \nu}^{+}}{\partial w} \theta(-w)+\frac{\partial h_{\mu \nu}^{-}}{\partial w} \theta(w) \\
\frac{\partial^{2} h_{\mu \nu}}{\partial w^{2}} & =\frac{\partial^{2} h_{\mu \nu}^{-}}{\partial w^{2}} \theta(-w)+\frac{\partial^{2} h_{\mu \nu}^{+}}{\partial w^{2}} \theta(w) \\
& +\left(\frac{\partial h_{\mu \nu}^{-}}{\partial w}-\frac{\partial h_{\mu \nu}^{+}}{\partial w}\right) \delta(w) .
\end{aligned}
$$

The equation (II.12) follows from (II.11) and (II.3) as a consequence of the fact that the terms $\partial K_{\mu}^{\nu} / \partial w$ and $\partial K / \partial w$ contain the delta function $\delta(w)$. However, in the fourth-order theory given by the action (I.2), the application of the continuity conditions (II.15)-(II.16) does not work. In order to discuss this let us first write down the field equations for the action (I.2) [15]:

$$
\begin{aligned}
P_{a b} & =\frac{\chi}{2} T_{a b}, \\
P^{a b} & =-\frac{1}{2} f g^{a b}+f_{X} R^{a b}+2 f_{Y} R^{c(a} R^{b)}{ }_{c}+2 f_{Z} R^{e d c(a} R^{b)} c d e \\
& +f_{X ; c d}\left(g^{a b} g^{c d}-g^{a c} g^{b d}\right)+\square\left(f_{Y} R^{a b}\right)+g^{a b}\left(f_{Y} R^{c d}\right)_{; c d} \\
& -2\left(f_{Y} R^{c(a}\right)_{;}{ }^{b)}-4\left(f_{Z} R^{d(a b) c}\right)_{; c d},
\end{aligned}
$$


where $f_{X}=\partial f / \partial X$ etc. The reason for not being the same continuity conditions (II.15)-(II.16) valid here is that the Riemann tensor

$$
R={ }^{(D-1)} R+\epsilon\left[2 h^{\mu \nu} \frac{\partial K_{\mu \nu}}{\partial w}+3 \operatorname{Tr}\left(K^{2}\right)-K^{2}\right],
$$

where $K \equiv K_{\mu}^{\mu}, \operatorname{Tr}\left(K^{2}\right) \equiv K^{\mu \nu} K_{\mu \nu}$, and appropriately, the Ricci tensor, and the Ricci scalar, as squared, give the terms

$$
\frac{\partial^{2} \gamma^{\mu \nu}}{\partial^{2} w} \frac{\partial K_{\mu \nu}}{\partial w}, \frac{\partial K_{\mu \nu}}{\partial w} \frac{\partial K^{\mu \nu}}{\partial w},\left(\frac{\partial K}{\partial w}\right)^{2}
$$

which are proportional to $\delta^{2}(w)$, For example, in the simple fourth-order gravity theory $f(X, Y, Z)=f(X)=R^{2}$ the field equations contain the term $f_{X}$, ww , which is proportional to $\delta^{2}(w)$.

As mentioned in the Introduction, the only "mysterious" cases which do not involve singular expressions are the Euler densities. In these cases the terms proportional to $\delta^{2}(w)$ exactly cancel. These theories have been studied by a number of people (e.g. [3, 4, 5, 6, 7, 8]) and their field equations are given by varying the action (I.1) with $\kappa_{1} \neq 0, \kappa_{1} \neq 0$ and $\kappa_{2} \neq 0$

$$
\begin{aligned}
G_{a b} & +\alpha H_{a b}+\Lambda g_{a b}=\kappa^{2} T_{a b} \\
G_{a b} & \equiv R_{a b}-\frac{1}{2} g_{a b} R \\
H_{a b} & \equiv 2\left(R_{a l m n} R_{b}^{l m n}-2 R_{a m b n} R^{m n}\right. \\
& \left.-2 R_{a m} R_{b}{ }^{m}+R R_{a b}\right)-\frac{1}{2} g_{a b} I^{(2)} .
\end{aligned}
$$

The "dangerous" terms appear in the "correction" $H_{a b}$ to the Einstein equations which is

$$
\begin{aligned}
& H^{\mu}{ }_{\nu}=4 \frac{\partial}{\partial w}\left\{K K_{\alpha}^{\mu} K_{\nu}^{\alpha}-K_{\alpha}^{\mu} K^{\alpha \beta} K_{\beta \nu}\right. \\
+ & \left.\frac{1}{2} K_{\nu}^{\mu} \operatorname{Tr}\left(K^{2}\right)-\frac{1}{2} K^{\mu}{ }_{\nu} K^{2}\right\} \\
+ & 4 \frac{\partial}{\partial w}\left\{-\delta^{\mu}{ }_{\nu} \frac{1}{2} K \operatorname{Tr}\left(K^{2}\right)+\delta^{\mu}{ }_{\nu} \frac{1}{3} \operatorname{Tr}\left(K^{3}\right)+\delta^{\mu}{ }_{\nu} \frac{1}{6} K^{3}\right\} \\
+ & 4\left(-{ }^{4} R^{\mu}{ }_{\alpha \nu}{ }^{\beta} \frac{\partial K^{\alpha}}{\partial w}-{ }^{4} R^{\alpha}{ }_{\nu} \frac{\partial K^{\mu}{ }_{\alpha}}{\partial w}-{ }^{4} R^{\alpha \mu} \frac{\partial K_{\nu \alpha}}{\partial w}\right) \\
+ & 4\left({ }^{4} R^{\mu}{ }_{\nu} \frac{\partial K}{\partial w}+\frac{1}{2}{ }^{4} R \frac{\partial K^{\mu}{ }_{\nu}}{\partial w}+\delta^{\mu}{ }_{\nu}{ }^{4} R^{\alpha \beta} \frac{\partial K_{\alpha \beta}}{\partial w}\right. \\
- & \left.\frac{1}{2} \delta^{\mu}{ }_{\nu}{ }^{4} R \frac{\partial K}{\partial w}\right)+\ldots,
\end{aligned}
$$

The junction conditions are obtained by using the continuity conditions (II.15)-(II.16) and read [5]

$$
\begin{aligned}
& 2 \alpha\left(3\left[J_{\mu \nu}\right]-[J] h_{\mu \nu}-2[P]_{\mu \rho \nu \sigma}[K]^{\rho \sigma}\right) \\
+ & {\left[K_{\mu \nu}\right]-[K] h_{\mu \nu}=-\kappa^{2} S_{\mu \nu} }
\end{aligned}
$$

where

$$
\begin{aligned}
P_{\mu \rho \nu \sigma} & =R_{\mu \rho \nu \sigma}+2 h_{\mu[\sigma} R_{\nu] \rho}+2 h_{\rho[\nu} R_{\sigma] \mu} \\
& +R h_{\mu[\nu} h_{\sigma] \rho}, \\
J_{\mu \nu} & =\frac{1}{3}\left(2 K K_{\mu \sigma} K_{\nu}^{\sigma}+K_{\sigma \rho} K^{\sigma \rho} K_{\mu \nu}-2 K_{\mu \rho} K^{\rho \sigma} K_{\sigma \nu}\right. \\
& \left.-K^{2} K_{\mu \nu}\right) .
\end{aligned}
$$

Two different ways to obtain the junction conditions have been applied so far. In Ref. [3] the limit $\lim _{w \rightarrow 0} \int_{-w}^{w}$ of the field equations (II.23)-(II.25) with the energymomentum tensor given by (II.3) has been taken. On the other hand, in Ref. [5] the appropriate Gibbons-Hawking 20] has been added to the Gauss-Bonnet action which cancelled normal derivatives of the metric variation. The form of the boundary term for this theory had been given earlier in Refs. 21, 22]. In fact, the exact form of the boundary term for the action (I.2) of this paper is not yet known and according to the claim of Ref. [21] it does not exist. According to Ref. 24 there is even an ambiguity of the choice of the boundary terms within Gauss-Bonnet brane world. A more general discussion of the boundary terms is given in Refs. [28, 29].

The discussion of the Gibbons-Hawking boundary term to the standard first-order Euler density (i.e. Ricci scalar) was also given, for example, in Ref. [25].

\section{A PROPOSAL TO FORMULATE CONSISTENT JUNCTIONS CONDITIONS IN THE FOURTH-ORDER THEORY ON THE BRANE}

Bearing in mind the roots of the irregularity of the field equations (II.20)-(II.21), and using the notation of [13, we suggest to define the following singular hypersurface of order three for which the metric derivatives and the extrinsic curvature on both sides of it are given by

$$
\begin{aligned}
h_{\mu \nu}^{-} & =h_{\mu \nu}^{+}, \\
h_{\mu \nu, w}^{-} & =h_{\mu \nu, w}^{+}, \quad K_{\mu \nu}^{-}=K_{\mu \nu}^{+}, \\
h_{\mu \nu, w w}^{-} & =h_{\mu \nu, w w}^{-}, \quad K_{\mu \nu, w}^{-}=K_{\mu \nu, w}^{+}, \\
h_{\mu \nu, w w w}^{-} & \neq h_{\mu \nu, w w w}^{+}, \quad K_{\mu \nu, w w}^{-} \neq K_{\mu \nu, w w}^{+}
\end{aligned}
$$

i.e., the metric and its first derivative are regular, the second derivative of the metric is continuous, but possesses a kink, the third derivative of the metric has a step function discontinuity, and as long as the fourth derivative of the metric on the brane produces the delta function contribution. On the other hand, according to 13 a singular hypersurface of order two is defined as

$$
\begin{aligned}
h_{\mu \nu}^{-} & =h_{\mu \nu}^{+}, \\
h_{\mu \nu, w}^{-} & =h_{\mu \nu, w}^{+}, \quad K_{\mu \nu}^{-}=K_{\mu \nu}^{+}, \\
h_{\mu \nu, w w}^{-} & \neq h_{\mu \nu, w w}^{-}, \quad K_{\mu \nu, w}^{-} \neq K_{\mu \nu, w}^{+},
\end{aligned}
$$

and it would describe the boundary surfaces characterized by jumps in the energy-momentum tensor (e.g. the 


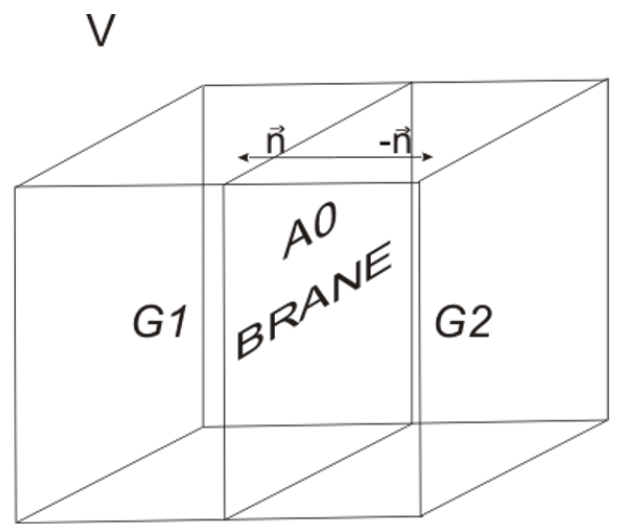

FIG. 1: A schematic picture illustrating the domains of integration used in derivation of the junction conditions. Here $V=G 1+G 2, A 1=\partial G 1+A 0$ and $A 2=\partial G 2-A 0$.

boundary surface separating a star from the surrounding vacuum). The physical interpretation of the singular hypersurfaces of order three is not so obvious, since it should be characterized by a jump of the first derivative of the energy-momentum tensor.

In order to carry on let us notice that the field equations (II.20)-(II.21) can be rewritten as

$$
\sqrt{-g} C_{a b} W_{; d}^{a b d}+\sqrt{-g} C_{a b} V^{a b}=\frac{\chi}{2} T^{a b} C_{a b} \sqrt{-g}(,
$$

where we have introduced is an arbitrary tensor field $C_{a b}$, and

$$
\begin{aligned}
W^{a b d} & =f_{X ; c}\left(g^{a b} g^{c d}-g^{(a c} g^{b) d}\right)+\left(f_{Y} R^{a b}\right)^{; d} \\
& +g^{a b}\left(f_{Y} R^{c d}\right)_{; c}-2\left(f_{Y} R^{d(a}\right)^{; b)}-4\left(f_{Z} R^{d(a b) c}\right)_{; c} \\
V^{a b} & =-\frac{1}{2} f g^{a b}+f_{X} R^{a b}+2 f_{Y} R^{c(a} R^{b)}{ }_{c} \\
& +2 f_{Z} R^{e d c(a} R^{b)}{ }_{c d e},
\end{aligned}
$$

contain third derivatives of the metric. In fact, $W^{a b d} ; d$ is proportional to $\delta(w)$, and the energy-momentum tensor $T_{a b}$ is given by (II.3). Now, we integrate both sides of (III.8) over the volume $V$ which contains the following parts (cf. Fig. 1): $G 1, G 2$ - are the left-hand-side and the right-hand-side bulk volumes which are separated by the brane, $A 1=\partial G 1+A 0, A 2=\partial G 2-A 0$ are the boundaries of these volumes, and $A 0$ is the brane which orientation is given by the direction of the normal vector $\vec{n}$. We have

$$
\begin{gathered}
\int_{G 1+G 2} \sqrt{-g} C_{a b} W_{; d}^{a b d} d \Omega \\
+\int_{G 1+G 2} \sqrt{-g} C_{a b} V^{a b} d \Omega=\int_{G 1+G 2} \frac{\chi}{2} T^{a b} C_{a b} \sqrt{-g} d \Omega,
\end{gathered}
$$

and so

$$
\begin{aligned}
& \int_{G 1+G 2} \sqrt{-g}\left(C_{a b} W^{a b d}\right)_{; d} d \Omega \\
- & \int_{G 1+G 2} \sqrt{-g} C_{a b ; d} W^{a b d} d \Omega \\
+ & \int_{G 1+G 2} \sqrt{-g} C_{a b} V^{a b} d \Omega \\
= & \int_{G 1} \frac{\chi}{2} T^{a b} C_{a b} \sqrt{-g} d \Omega+\int_{G 2} \frac{\chi}{2} T^{a b} C_{a b} \sqrt{-g} d \Omega \\
+ & \int_{A 0} \frac{\chi}{2} S^{a b} C_{a b} \sqrt{-\gamma} d \sigma .
\end{aligned}
$$

Using the Gauss theorem first

$$
\int_{G 1+G 2} \sqrt{-g}\left(C_{a b} W^{a b d}\right)_{; d} d \Omega=\int_{A 1+A 2} \sqrt{-\gamma} C_{a b} W^{a b d} n_{d} d \sigma,
$$

we then integrate in the limit $V \rightarrow A 0$ (which in Gaussian normal coordinates (II.4) corresponds to the limit $\left.\lim _{w \rightarrow 0} \int_{-w}^{w}\right)$ to obtain

$$
\int_{A 0} \sqrt{-\gamma} C_{a b}\left\{[W]^{a b d} n_{d}-\frac{\chi}{2} S^{a b}\right\}=0,
$$

where $[W]^{a b d}=W^{a b d+}-W^{a b d-}$. Since the tensor $C_{a b}$ is arbitrary one can then conclude that

$$
[W]^{a b d} n_{d}-\frac{\chi}{2} S^{a b}=0,
$$

and these are exactly the junction conditions for the theory under study.

For example, in $f(X, Y, Z)=f(X)=f(R)$ theory in $D=5$ dimensions with metric

$$
\begin{aligned}
d s^{2} & =-d t^{2} \\
& +a^{2}(t, w)\left[d r^{2}+r^{2}\left(d \Theta^{2}+\sin ^{2} \Theta d \phi^{2}\right)\right]+d w^{2}
\end{aligned}
$$

they give

$$
\begin{aligned}
{\left[a^{\prime \prime \prime}\right] } & =\frac{\chi}{2} a_{0} p_{0}, \\
p_{0} & =\rho_{0},
\end{aligned}
$$

where $(\ldots)^{\prime}=\partial / \partial w, a_{0}=a(w=0)$ and the brane energy-momentum tensor $S_{\mu}^{\nu}=\left(-\rho_{0}, p_{0}, p_{0}, p_{0}\right)$.

\section{EQUIVALENT SECOND-ORDER THEORY APPROACH}

It is known that the fourth-order gravity theory

$$
S=\chi^{-1} \int d^{D} x \sqrt{-g} f(R)
$$

is equivalent to a second-order theory with the action 18

$$
S=\chi^{-1} \int d^{D} x \sqrt{-g}\left[f^{\prime}(Q)(Q-R)+f(Q)\right]
$$


of which equation of motion is just $Q=R$, provided $f^{\prime \prime}(Q) \neq 0$. In this approach $f^{\prime}(Q)$ may be interpreted as an extra scalar field $\phi=f^{\prime}(Q)$.

Similar approach can also be used to the fourth-order gravity of the function of Gauss-Bonnet term

$$
S=\chi^{-1} \int d^{D} x \sqrt{-g} f\left(R_{G B}\right)
$$

by considering the second-order theory action [19]

$$
S=\chi^{-1} \int d^{D} x \sqrt{-g}\left[f^{\prime}(A)\left(R_{G B}-A\right)+f(A)\right]
$$

The variation of this action with respect to $A$ gives the equation of motion $A=R_{G B}$, provided $f^{\prime \prime}(A) \neq 0$ and $\psi=f^{\prime}(A)$ is interpreted as an extra scalar field.

A step ahead can be made by considering more general fourth-order theory [26, 27]

$$
S=\chi^{-1} \int d^{D} x \sqrt{-g} f\left(g_{a b}, R_{a b}\right)
$$

which can be made equivalent to the second-order theory provided that one introduces the tensor field

$$
k^{a b}=k^{a b}\left(g_{a b}, R_{a b}\right)=\frac{\partial f}{\partial R_{a b}},
$$

which reduces the appropriate field equations to the second-order and eventually transforms it into the Einstein theory with some new metric tensor.

We follow these considerations by noticing that the theory given by the action (I.2) is a special case of a more general theory with the action of the form

$$
S_{G}=\chi^{-1} \int_{M} d^{D} x \sqrt{-g} f\left(g_{a b}, R_{a b c d}\right) .
$$

In fact, it is enough to consider the theory (IV.7) in order to get all the previous actions (IV.1), IV.3) and (IV.5) as well as the theory with the linear combinations of curvature terms $f(X, Y, Z)=a X^{2}+b Y+c Z$, as in Ref. [17.

In general, the action (IV.7) again gives immediately the fourth-order field equations, but there exist a possibility to formulate it in an equivalent way by using the action of the following form

$$
\begin{aligned}
S_{I} & =\chi^{-1} \int_{M} d^{D} x \sqrt{-g}\left\{\frac{\partial f\left(g_{a b}, \phi_{a b c d}\right)}{\partial \phi_{g h i j}}\left(R_{g h i j}-\phi_{g h i j}\right)\right. \\
& \left.+f\left(g_{a b}, \phi_{c d e f}\right)\right\}
\end{aligned}
$$

where $\phi_{a b c d}$ is the tensor field which is independent of $g_{a b}$. The variation of the action IV.8 with respect to $\phi_{a b c d}$ gives the equation of motion which is simply

$$
R_{g h i j}=\phi_{g h i j}
$$

provided that the determinant

$$
\operatorname{det}\left[\frac{\partial^{2} f\left(g_{a b}, \phi_{a b c d}\right)}{\partial \phi_{g h i j} \partial \phi_{k l m n}}\right] \neq 0 \text {. }
$$

The condition IV.10 holds for the fourth-order theory with the action (I.2), too. This means that $(\overline{I .2})$ and IV.8 are the equivalent actions. Introducing the tensor

$$
H^{g h i j} \equiv \frac{\partial f\left(g_{a b}, \phi_{a b c d}\right)}{\partial \phi_{g h i j}},
$$

and then varying the full action $S=S_{I}+S_{m}$ with respect to both $H^{a b c d}$ and $g_{a b}$, we get the field equations in the following form:

$$
\begin{aligned}
R_{g h i j} & =-\frac{\partial V\left(g_{a b}, H^{c d e f}\right)}{\partial H^{g h i j}} \\
\frac{1}{2} g^{a b} f & +\frac{\partial f}{\partial g_{a b}}+H^{b e c d} \phi_{e c d}^{a}\left(g_{a b}, H^{k l m n}\right)+ \\
& +\left\{A^{(a b) c d}\right\}_{; d c}=-\frac{\chi}{2} T^{a b}
\end{aligned}
$$

where

$$
\begin{aligned}
A^{a b c d}=\frac{1}{2}\left\{H^{a c d b}\right. & +H^{a b d c}-H^{c b d a}-H^{a c b d}+ \\
& \left.-H^{a b c d}+H^{c b a d}\right\}
\end{aligned}
$$

and

$$
\begin{gathered}
V\left(g_{a b}, H^{c d e f}\right)= \\
-H^{h g i j} \phi_{g h i j}\left(g_{a b}, H^{c d e f}\right)+f\left(g_{a b}, \phi_{k l m n}\left(g_{a b}, H^{c d e f}\right)\right)
\end{gathered}
$$

In fact, the possibility to express the fields $\phi_{a b c d}$ as a function of $g_{a b}$ and $H^{c d e f}$ is guaranteed by the condition IV.10.

\section{JUNCTION CONDITIONS AND THEIR CORRESPONDENCE IN THE EQUIVALENT SECOND-ORDER THEORY}

The tensor

$$
M^{a b}=\frac{1}{2} g^{a b} f+\frac{\partial f}{\partial g_{a b}}+H^{b e c d} \phi_{e c d}^{a}\left(g_{a b}, H^{k l m n}\right)
$$

is a function of the fields $g_{a b}$ and $H^{a b c d}$ only, and it does not give any contribution to the junction conditions. This is because $H^{a b c d}$ are continuous on the brane, their first normal derivatives have a jump, and their second normal derivatives are proportional to the $\delta(y)$ function on the brane. Performing the same type of calculation as in the Section III the relation (IV.13) gives the junction conditions in the form:

$$
\left[A_{; d}^{(a b) c d}\right] n_{c}=-\frac{\chi}{2} S^{a b} .
$$

Assuming that IV.9 is fulfilled, and that

$$
f\left(g_{a b}, \phi_{a b c d}\right)=f\left(\phi_{a b}^{a b}, \phi_{a c b}{ }^{c} \phi_{c}^{a c b}, \phi_{a b c d} \phi^{a b c d}\right),
$$

we have

$$
\begin{aligned}
{\left[A_{; d}^{(a b) c d}\right] n_{c} } & =\left[A_{; c}^{(a b) c d}\right] n_{d}=\left[-\left\{f_{X ; c}\left(g^{a b} g^{c d}-g^{c(a} g^{b) d}\right)\right.\right. \\
& +\left(f_{Y} R^{a b}\right)^{; d}+g^{a b}\left(f_{Y} R^{c d}\right)_{; c} \\
-2\left(f_{Y} R^{d(a}\right)^{; b)} & \left.\left.-4\left(f_{Z} R^{d(a b) c}\right)_{; c}\right\}\right] n_{d}=-\left[W^{a b d}\right] n_{d} .
\end{aligned}
$$


This means that the junction conditions V.1 in the second-order theory (IV.8) are completely equivalent to the juction conditions (III.14) in the fourth-order theory (I.2).

The equation (IV.12), which corresponds to (IV.9) implies that Riemann tensor is continuous on the brane, since the fields $\phi_{a b c d}$ are regular functions of continuous fields $H^{a b c d}$ and $g_{a b}$. This, together with the GaussCodazzi equations (II.6) imply the following junction conditions:

$$
\begin{array}{r}
{\left[K_{a b}\right]=0} \\
{\left[\mathcal{L}_{\vec{n}} K_{a b}\right]=0,}
\end{array}
$$

where $\mathcal{L}_{\vec{n}}$ is a Lie derivative of $H^{a(e f) d}$ along the vector field $\vec{n}$. (Vector field $\vec{n}$ is a normalized vector field tangent to the Gaussian normal coordinate $w$ ). The equations (V.4) reconstruct the assumptions (V.1).

Bearing in mind the formulas above, equations (V.1) can be reduced to:

$$
n_{c} n_{d}\left[\mathcal{L}_{\vec{n}} A^{(a b) c d}\right]=-\frac{\chi}{2} S^{a b},
$$

where

$$
\begin{aligned}
A^{a b c d} & =f_{X}\left(g^{a d} g^{c b}-g^{c d} g^{b a}\right) \\
& +f_{Y}\left(2 R^{a d} g^{b c}-R^{c d} g^{b a}-R^{b a} g^{c d}\right) \\
& +4 f_{Z} R^{a c b d} .
\end{aligned}
$$

In Gaussian normal coordinates the conditions (V.4) and (V.5) read as

$$
\begin{aligned}
{\left[K_{a b, w}\right] } & =0, \\
n_{c} n_{d}\left[A_{, w}^{(a b) c d}\right] & =-\frac{\chi}{2} S^{a b} .
\end{aligned}
$$

In a specific example of $f(X, Y, Z)=Z$ theory for the metric (III.15), the equations (V.4) and (V.5) give:

$$
\begin{aligned}
8 \frac{\left[a^{\prime \prime \prime}\right]}{a_{0}} & =\frac{\chi}{2} \rho_{0}, \\
p_{0} & =-\rho_{0} .
\end{aligned}
$$

\section{SUMMARY}

In this paper we have considered the junction conditions for the fourth-order brane world given by the action which is a general function of the curvature invariants $R, R_{a b} R^{a b}$, and $R_{a b c d} R^{a b c d}$. We imposed the regularity conditions on the metric tensor which was taken to be of the class $C^{2}$ functions of the coordinates. Explicitly, it means that the metric and its first derivative are regular at the brane, while the second derivative has a kink, the third derivative of the metric has a step function discontinuity, and the fourth derivative of the metric gives the delta function contribution to the field equations. In terms of the seminal notation given first by Israel [13, these conditions describe the singular hypersurfaces of order three. The junction conditions which we obtained are quite generic and they may be applied to some special cosmological framework of interest.

As an alternative which allows less restrictive regularity conditions, we considered the reduction of the fourthorder theory to a second-order theory by applying an extra tensor field. We then formulated the junction conditions within such a theory and showed that they were equivalent to the previously obtained fourth-order theory junction conditions.

In the previously considered cases in the literature mainly the Eulerian density brane worlds were studied 3, 4, 5, 6, 7, 8, 9, 11. The only non-Eulerian density cases were investigated in Refs. [16] and [17, where the theories with at most a linear combination the curvature invariants of the form $f(X, Y, Z)=a X^{2}+b Y+c Z$ ( $a, b, c=$ const.) were considered. In these references the junction conditions were obtained after transforming such theories into the equivalent second-order theories. In fact, we made one step further, by suggesting junction conditions for the brane world which allows a general function of curvature invariants $f(X, Y, Z)$. Also, in one of our approaches to the problem of junction conditions we do not reduce the fourth-order action into the second-order action, but suggest junction conditions to work in the fourth-order theory, though in the case of the so-called singular hypersurfaces of order three only [13.

We hope that our calculations will allow us to study some cosmological applications of the general fourthorder gravity on the brane $(\mathrm{I} .2)$ in the following papers.

\section{ACKNOWLEDGEMENTS}

We thank Andrei Barvinsky, Salvatore Capozziello, Tomasz Denkiewicz, Krzysztof Meissner and David Wands for discussions. M.P.D. acknowledges the support of the Polish Ministry of Science and Higher Education grant No 1 P03B 04329 (years 2005-2007).
[1] L. Randall and R. Sundrum, Phys. Rev. Lett., 83, 3370 (1999); L. Randall and R. Sundrum, ibidem, 83, 4690 (1999).

[2] M. Visser, Phys. Lett. B159, 22 (1985); N. ArkaniHamed, S. Dimopoulos, and G. Dvali, Phys. Lett. B516,
70 (1998); I. Antoniadis, N. Arkani-Hamed, S. Dimopoulos, G. Dvali, Phys. Lett. B436, 257 (1998); N. ArkaniHamed, S. Dimopoulos, and G. Dvali, Phys. Rev. D59, 086004 (1999); P. Binétruy, C. Deffayet and D. Langlois, Nucl. Phys. B565, 269 (2000); P. Binétruy, C. Def- 
fayet and D. Langlois, Phys. Lett. B477, 285 (2000); M. Sasaki, T. Shiromizu and K. Maeda, Phys. Rev. D62, 024008 (2000); T. Shiromizu, K. Maeda, and M. Sasaki, Phys. Rev. D62, 024012 (2000); S. Mukhoyama, T. Shiromizu and K. Maeda, Phys. Rev. D62, 024028 (2000); A.N. Aliev and A.E. Gümrükçüg̃lu, Class. Quantum Grav. 21, 5081 (2004); D. Cremades, L.E. Ibanez and F. Marchesano, Nucl. Phys. B 643, 93 (2002); C. Kokorelis, Nucl. Phys. B 677, 115 (2004).

[3] N. Deruelle and T. Doležel, Phys. Rev. D62, 103502 (2000).

[4] C. Charmousis, J.F. Dufaux, Class. Quantum Grav. 19, 4671 (2002).

[5] S.C. Davis, Phys. Rev. D 67, 024030 (2003).

[6] J.F. Dufaux, J.E. Lidsey, R. Maartens, and M. Sami, Phys. Rev. D 70, 083525 (2004).

[7] J.E. Lidsey, Ann. Phys. (Leipzig) 15, 277 (2006).

[8] H. Maeda, V. Sahni, Yu. Shtanov, gr-qc/0708.3287.

[9] P.S. Apostopoulos et al., hep-th/0708.0469.

[10] D. Lovelock, J. Math. Phys. 12, 498 (1971).

[11] K.A. Meissner and M. Olechowski, Phys. Rev. Lett. 86, 3708 (2001).

[12] S.W. Hawking, G.F.R. Ellis, The Large-scale Structure of Space-time, (Cambridge Univ. Press, 1999) .

[13] W. Israel, Nuovo Cimento B 44, 1 (1966).

[14] M. Visser, Lorentzian Wormholes (Springer-Verlag, 1996).

[15] T. Clifton and J.D. Barrow, Phys. Rev. D 72, 123003 (2005); T. Clifton and J.D. Barrow, Class. Quantum Grav. 23, 2951 (2006).

[16] M. Parry, S. Pichler, and D. Deeg, JCAP 0504, 014 (2005).

[17] S. Nojiri and S.D. Odintsov, JHEP 0007, 049 (2000); S. Nojiri, S.D. Odintsov and S. Ogushi, Phys. Rev. D 65, 023521 (2001).

[18] A.A. Starobinsky, Phys. Lett. B 91, 99 (1980); G. Magnano and L.M. Sokołowski, Phys. Rev. D 50, 5039 (1994);
T.P. Sotiriou, Class. Quantum Grav. 23, 5117 (2006); T. Chiba, T.L. Smith, and A.L. Erickcek, Phys. Rev. D 75, 043516 (2007); G.J. Olmo, Phys. Rev. Lett. 98, 061101 (2007); G.J. Olmo, Phys. Rev. D 75, 023511 (2007); S. Capozziello, V.F. Cardone, and A. Troisi, Phys. Rev. D 71, 043503 (2005); S. Capozziello, S. NOjiri, S.D. Odintsov, and A. Troisi, Phys. Lett. B 639, 135 (2006); T. Chiba, Phys. Rev. D 75, 043516 (2007); S. Capozziello and R. Garatini, Class. Quantum Grav. 24, 1627 (2007); L. Amendola, D. Polarski, and S. Tsujikawa, Phys. Rev. Lett. 98, 131302 (2007); T. Faulkner et al., astro-ph/0612569.

[19] S. Nojiri, S.D. Odintsov, Phys. Lett. B 631, 1 (2005); S. Nojiri, S.D. Odintsov, O.G. Gorbunova, J. Phys. A 39, 6627 (2006); G. Cognola et al., Phys. Rev. D 73, 084007 (2006); B. Li, J.D. Barrow, D.F. Mota, Phys. Rev. D 76, 044027 (2007); S.C. Davis, hep-th/0709.4453.

[20] G.W. Gibbons and S.W. Hawking, Phys. Rev. D 15, 2752 (1977).

[21] T.S. Bunch, Journ. Phys. A 14, L139 (1981).

[22] F. Müller-Hoissen, Phys. Lett. B 163, 106 (1985); R.C. Myers, Phys. Rev. D 36, 392 (1987).

[23] P.D. Mannheim, Brane Localized Gravity (World Scientific, 2005).

[24] S. Nojiri and S.D. Odintsov, Gen. Rel. Grav. 37, 1419 (2005).

[25] E. Papantopoulos, Lect. Notes Phys. 592, 458 (2002).

[26] A. Jakubiec and J. Kijowski, Phys. Rev. D 37, 1406 (1988).

[27] J.D. Barrow and S. Hervik, Phys. Rev. D 73, 023007 (2006); ibidem, 74, 124017 (2006).

[28] A.D. Barvinsky and S.N. Solodukhin, Nucl. Phys. B 479, 305 (1996).

[29] T.P. Sotiriou and S. Liberati, Phys. Rev. D 74, 044016 (2006). 